\title{
Búsqueda de la calidad del aprendizaje mediante herramientas de inteligencia artificial ( $\cdots$ )
}

\author{
Lucas Dima ', Rafael Urdaneta`, Gabriel Sosa`, Gabriela Esperón`, \\ Daniela López De Luise ••
}

\section{Resumen}

Este trabajo expone, mediante el uso de técnicas de minería de datos e inteligencia artificial, una alternativa para determinar posibles factores ajenos a los académicos que puedan interferir de manera positiva o negativa en la calidad del aprendizaje de estudiantes universitarios del área de Matemática. Para tal fin se relevó una serie de datos socioeconómicos considerados relevantes por especialistas, sociólogos y pedagogos, incorporando adicionalmente una serie de evaluaciones prediseñadas con aspectos conceptuales, algebraicos y de modelización. Se emplea el método de clasificación bietápico el cual es una herramienta de exploración diseñada para descubrir las agrupaciones naturales (o conglomerados) de un conjunto de datos que, de otra manera, no sería posible detectar. A partir de este agrupamiento se establecerá la base para la elaboración de reglas que alimentarán al sistema experto para la formulación de conclusiones y recomendaciones para los estudiantes y docentes.

Palabras clave: Algoritmos de agrupamiento, arquitectura, problemas en el aprendizaje, minería de datos, sistemas expertos.

Fecha de Recepción: agosto 2012 | Fecha de aceptación: octubre 2012

- Investigadores del AlGroup, Universidad de Palermo, Argentina

-• Directora del AlGroup, Universidad de Palermo, Argentina

-• Este trabajo fue aprobado y presentado en el Congreso TRIC $\vee$ organizado por IEEE CIS (Computational Intelligence Society) Argentina que se desarrolló en el marco del IEEE Argencon 2012 del 13 al 15 de junio de 2012 en la UNC. 


\section{Abstract}

This paper presents an alternative to determine possible factors beyond academic ones that may interfere positively or negatively on the quality of learning of college students in Mathematics, by employing data mining techniques and artificial intelligence. For this purpose, a set of socio-economic data considered relevant by specialists, sociologists and educators were surveyed. Additionally series of pre designed evaluations containing conceptual, algebraic and modeling features were incorporated. To analyze the data, the two-step classification algorithm was performed. This algorithm is a scan tool designed to reveal natural groupings (or clusters) of a dataset that would otherwise not be apparent. This cluster analysis will provide the basis for the rules development that will feed an Expert System for creating conclusions and recommendations for students and teachers.

Keywords: quality of learning, data mining, classification algorithm, expert systems. 


\section{Introducción}

En los últimos años se ha intensificado la fusión entre la ciencia computacional y el dominio de la educación. Esto ha sido posible por el interés de las instituciones $\mathrm{y}$ de los investigadores por mejorar el rendimiento de docentes y alumnos en el proceso de aprendizaje.

En este punto, las técnicas y paradigmas de las herramientas como la Inteligencia Artificial aparecen como interesantes alternativas a los problemas que surgen al intentar introducir las computadoras para apoyar las diferentes estrategias de aprendizaje.

Si se considera la dimensión formativa que debería tener el proceso de evaluación, resulta de fundamental importancia aludir al concepto de "retroalimentación", la cual supone la identificación, en mayor o menor medida, de las fortalezas y debilidades en el conocimiento y las habilidades de los estudiantes, así como la identificación de las causas que dificultan dicho proceso[1].

Es aquí donde distintas técnicas de inteligencia artificial tales como algoritmos de agrupamiento y sistemas expertos podrían constituirse en poderosas herramientas tanto para identificar posibles causas vinculadas a un deficiente progreso en el desempeño académico y el proceso de aprendizaje, como para proveer una retroalimentación adecuada que a partir de recomendaciones efectuadas por el sistema permita a los alumnos revisar y corregir sus propios procesos. Por ejemplo, los sistemas expertos ofrecen la posibilidad de diagnosticar, depurar y corregir las producciones de los estudiantes en un área particular del conocimiento ya que permiten determinar el nivel cognoscitivo de los alumnos y ayudar a desarrollar y mejorar sus habilidades [2].

Adicionalmente, la implementación de un sistema como el proyecto Einstein, que aquí se propone, también brindaría información a los docentes para el diseño de situaciones didácticas que permitan mejorar la calidad del aprendizaje[3]. Este proyecto se desarrolla en el marco del AIGroup de la Universidad de Palermo.

El resto del trabajo se organiza como sigue: la sección 2 describe la arquitectura del sistema; en la sección 3 se describe cada uno de los módulos componentes del mismo; en la sección 4 se presenta los primeros resultados analizados en base a una encuesta de 32 alumnos inscriptos en varios cursos de Algebra Lineal; en la sección 5 se presentan las conclusiones y los trabajos futuros a desarrollar.

\section{Arquitectura}

El sistema está siendo desarrollado en el lenguaje orientado a objetos Java versión 1.6 (TM)[4]. La elección del mismo se debe al alto grado de portabilidad, 
es decir que sus aplicaciones son independientes del sistema operativo en el que se ejecuten. Otras características importantes son su madurez, la gran cantidad de herramientas ya desarrolladas y de aportes existentes por parte de la comunidad.

El sistema toma como entrada los datos de un grupo de estudiantes junto con la definición de los mismos. Esta definición consta de dos grupos: definición de la estructura de las respuestas (Tabla I) y definición de datos socioeconómicos (Tabla II).

Tabla I: Definicion de la estructura de las respuestas.

\begin{tabular}{|l|l|l|l|}
\hline VARIABLE & TIPO & UTILIZACIÓN & EJEMPLO \\
\hline $\begin{array}{l}\text { Identificador } \\
\text { único }\end{array}$ & Alfanumérico & $\begin{array}{l}\text { Identificador interno del } \\
\text { sistema }\end{array}$ & PRAX412 \\
\hline $\begin{array}{l}\text { Nombre de } \\
\text { la clase de la } \\
\text { respuesta }\end{array}$ & Alfanumérico & $\begin{array}{l}\text { Corresponde al nombre } \\
\text { completo de clase Java que } \\
\text { identifica al tipo de respuesta }\end{array}$ & $\begin{array}{l}\text { edu.palermo. } \\
\text { einstein.answers. } \\
\text { pleOptionNotOrderedAnswer }\end{array}$ \\
\hline $\begin{array}{l}\text { Estrategia de } \\
\text { análisis de la } \\
\text { respuesta [5] }\end{array}$ & Alfanumérico & $\begin{array}{l}\text { Indica la estrategia utilizada } \\
\text { para realizar el análisis de los } \\
\text { datos. Corresponde al nombre } \\
\text { completo de la clase Java }\end{array}$ & $\begin{array}{l}\text { edu.palermo.einstein.parser. } \\
\text { strategy.CsvParserStrategy }\end{array}$ \\
\hline
\end{tabular}

Tabla II: Definición de datos socioeconómicos

\begin{tabular}{|l|l|l|}
\hline VARIABLE & TIPO DE DATO & EJEMPLO \\
\hline Identificador único & Alfanumérico & UPAL2001004128 \\
\hline Fecha de nacimiento & Fecha & $1983-05-18$ \\
\hline Recurrente de la asignatura & Booleano & Falso \\
\hline Casado & Booleano & Verdadero \\
\hline Trabaja & Booleano & Verdadero \\
\hline Cantidad de hijos & Numérico & 0 \\
\hline Quien paga los estudios & Lista de opciones & Uno mismo \\
\hline Relación con la profesión & Booleano & Verdadero \\
\hline Cantidad de horas diarias de trabajo & Numérico & 8 \\
\hline Cantidad de horas semanales de estudio & Numérico & 40 \\
\hline Viene de otra universidad & Booleano & Falso \\
\hline
\end{tabular}


Los datos y la definición de los mismos son ingresados al módulo de interpretación el cual genera los objetos que representan a un individuo y que contiene sus datos socioeconómicos tanto como las respuestas a la evaluación realizada. Estas representaciones se van almacenando en una base de datos para el posterior análisis por el módulo inteligente. Este último es el encargado de implementar herramientas de minería de datos, mediante las cuales:

- Se accede a los objetos anteriormente mencionados.

- Se aplican los algoritmos de agrupamiento y se obtienen los conglomerados.

- Se formulan las reglas del sistema experto en función de las características predominantes en cada uno de los conglomerados.

- Las reglas permitirán que el sistema brinde recomendaciones a los usuarios acerca de las habilidades matemáticas que se deberían revisar con el fin de afianzarlas, modificarlas o mejorarlas.

- El módulo inteligente realizará la devolución de los resultados al usuario a través de una interfaz gráfica a la vez que almacenará nuevamente los datos en el Almacenamiento Persistente.

La figura 1 muestra el diagrama de proceso.

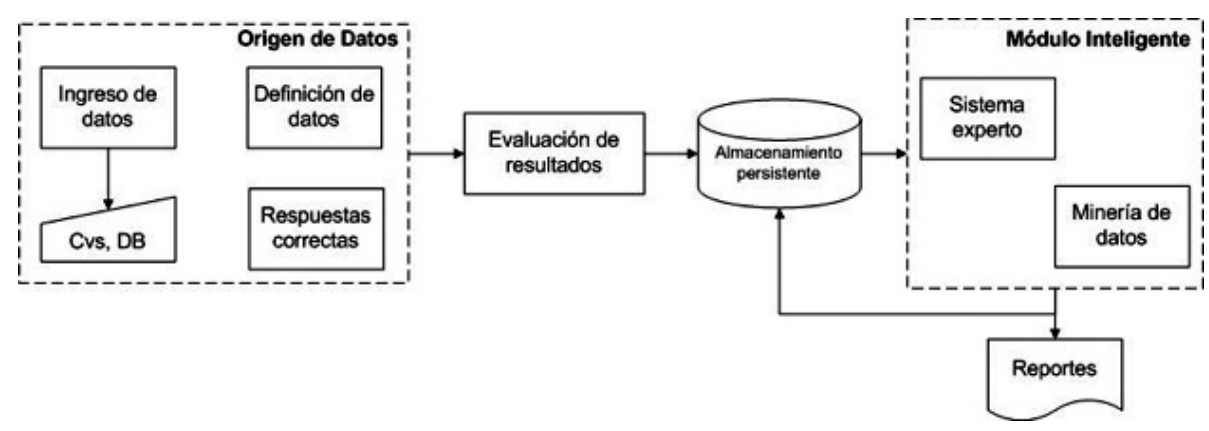

Fig. 1: Diagrama de proceso.

El funcionamiento del sistema en orden cronológico se puede ver en el siguiente diagrama de secuencia (Fig. 2) 


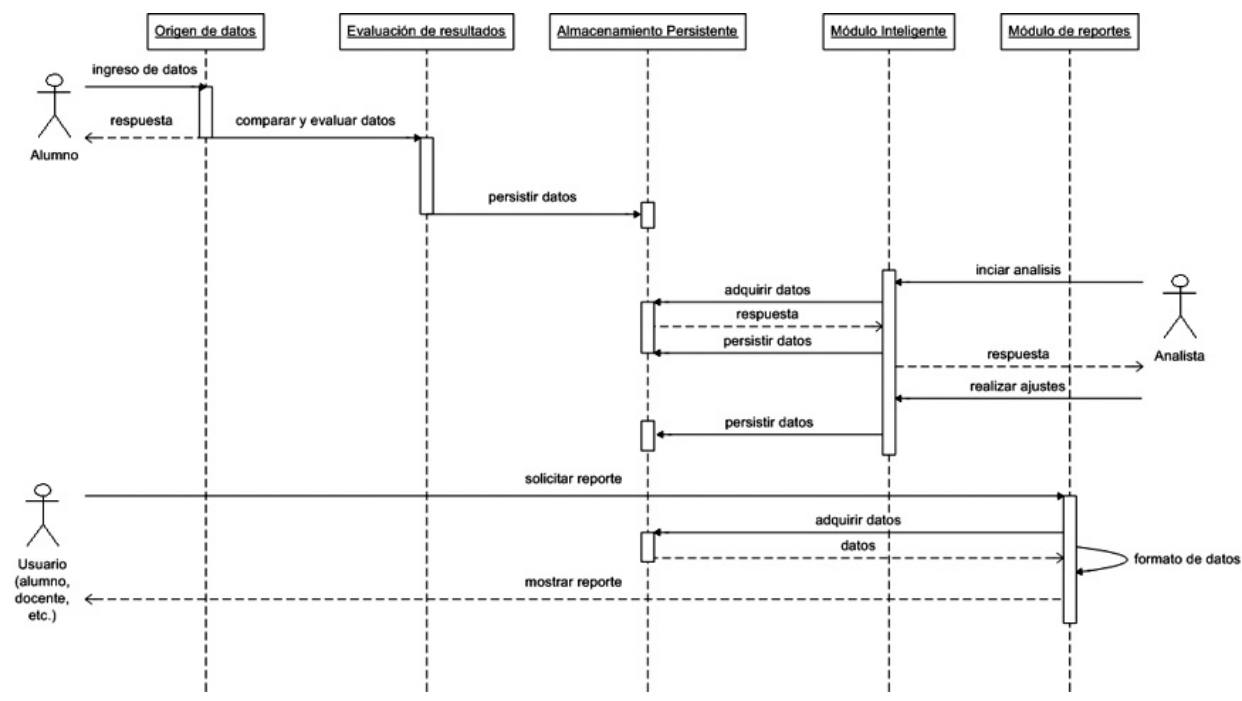

Fig. 2: Diagrama de secuencia.

\section{Descripción de los componentes}

\section{Módulo De Origen De Datos}

El módulo Origen de datos está formado por tres componentes: Ingreso de Datos,

\section{Definición de Datos y Respuestas Correctas.}

1) Ingreso De Datos: El sistema toma como entrada los datos de un grupo de estudiantes de una asignatura dada y las respuestas de un conjunto de ejercicios. Este proceso consta de dos partes. La primera es la carga de los datos socioeconómicos de cada una de las personas pertenecientes al grupo a evaluar y la segunda es la resolución del conjunto de ejercicios.

Para los datos socioeconómicos se escogieron una serie de variables relevantes que luego serán evaluadas por el resto de los componentes del sistema. La figura 3 (a) muestra el módulo de ingreso de datos. 


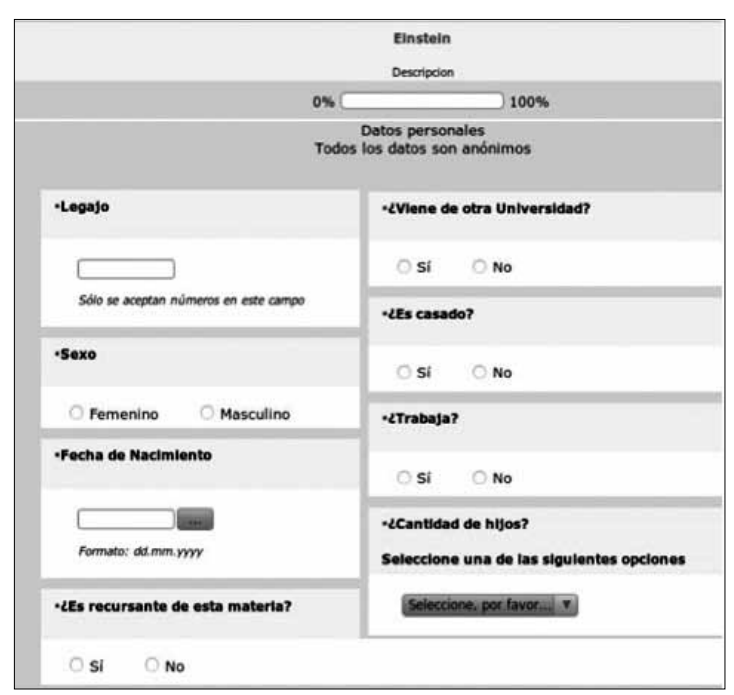

Fig. 3 (a): Módulo de Ingreso de datos

La segunda parte de los datos de entrada corresponde a las respuestas de un conjunto de ejercicios por parte cada uno de los individuos. En cuanto a la parte técnica y en virtud de mantener todo el proyecto utilizando software código abierto (open source) con licenciamiento GNU GPL se eligió para la carga de datos la aplicación web Limesurvey[6], que es completamente configurable y posee una interfaz clara y concisa, indispensable para que el ingreso de datos no entorpezca el proceso, y sea lo más sencillo y rápido posible para el individuo a examinar. Se calcula que con un conjunto de ejercicios estándar cada individuo puede tardar entre 5 y 10 minutos para cargar sus datos y sus respuestas.

También se utilizó esta herramienta incorporar la resolución de los ejercicios matemáticos, presentando una forma simple y amistosa para ingresar las sus resoluciones.

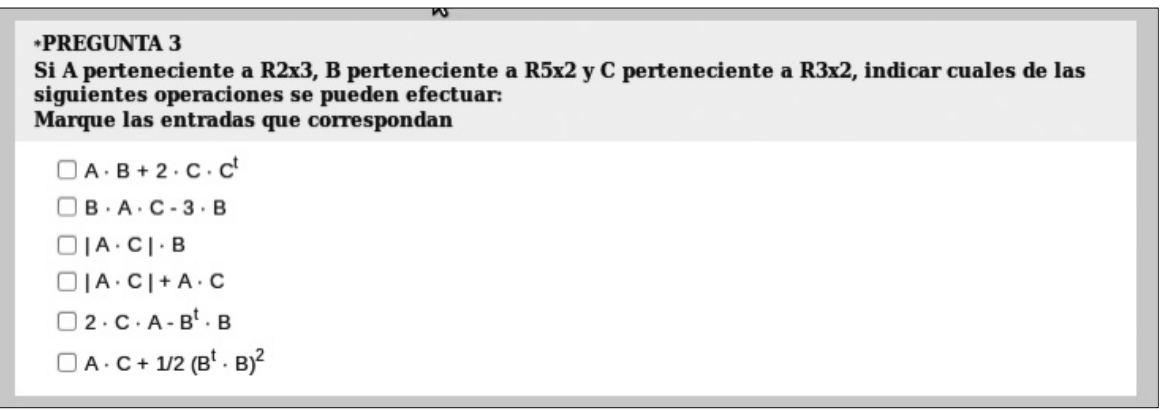

Fig. 3 (b): Módulo de Ingreso de datos - Ejercicios matemáticos 
El hecho de que la carga de datos se realice a través de una aplicación web permite que se puedan evaluar muestras independientemente de su locación geográfica, logrando un buen complemento para entornos de educación a distancia.

Junto con los datos de entrada, se incluirá una clave única por cada alumno que permitirá la realización de un seguimiento longitudinal del desempeño de este individuo.

Uno de los puntos clave de la arquitectura del proyecto es que permite interactuar con datos en cualquier formato. Para lo cual se decidió que los mismos se encuentren separados de sus meta-datos [7].

2) Definición de Datos: Este componente es el encargado de interpretar la definición de los datos propiamente dichos y lograr que el sistema vea de manera transparente los datos que requiere procesar. La definición de los datos se encuentra en un archivo modelado en formato YAML [8], el cual contiene lo necesario para interpretar los datos de manera adecuada. Se optó por este formato ya que el mismo se encuentra ampliamente adoptado y es de fácil interpretación para el operador del sistema. Este enfoque permite que la definición de los datos pueda ser modificada sin realizar cambios en el núcleo del sistema, ya que está almacenada de forma externa en un archivo de texto plano. También permite un ágil manejo por parte del administrador y le otorga flexibilidad y versatilidad a todo el sistema.

3) Respuestas Correctas: Contiene las respuestas correctas que se utilizarán para contrastar los resultados de los ejercicios que realizan los alumnos.

\section{B. Módulo de Evaluación de Resultados}

Este módulo recibe los archivos procesados por el Módulo de Origen de datos y se encarga de realizar la comparación entre las respuestas generadas por los estudiantes y el conjunto de respuestas correctas.

En esta primera etapa se han considerado tres tipos de respuestas para los ítems:

- Respuesta simple cerrada: donde el estudiante debe seleccionar una opción dentro de las listadas.

- Respuesta múltiple no ordenada: permite seleccionar varias opciones entre las listadas y donde el orden dado de las respuestas no es considerado.

- Respuesta múltiple ordenada: permite seleccionar varias respuestas y debe tener en consideración el orden dado de las respuestas.

En este punto los registros de los estudiantes se encuentran en un formato abstracto, conteniendo la información socioeconómica de cada uno de ellos, como así también las respuestas de cada uno de los ítems de los ejercicios. 
Los registros serán almacenados para luego ser procesados por el Módulo Inteligente.

\section{Módulo Inteligente}

Se encuentra dividido en dos componentes, el componente de Minería de Datos y el Sistema Experto.

El componente de Minería de Datos, obtendrá los datos de los resultados de las evaluaciones procesadas y persistidas por el módulo de Evaluación de Resultados (Parser) y seguidamente ejecutará una técnica de minería de datos, mediante la cual se clasifica la población analizada, agrupando los miembros en grupos con características similares y a la vez, separándolos en base a las características inter grupales.

En este componente se integrarán las respuestas de los alumnos a los ejercicios y sus correspondientes datos socioeconómicos. El estudio exhaustivo de las características de los conglomerados permitirá la elaboración de perfiles que posibilitarán la creación de las reglas que empleará el Sistema Experto ¡Error! No se encuentra el origen de la referencia.-[9].

Cada grupo generado, representa a un conjunto de alumnos con características similares y a su vez cada grupo se distingue de otro por su heterogeneidad entre ellos. De esta manera obtenemos grupos con características particulares en base a las variables definidas en el primer módulo del sistema. A efectos de los estudios realizados y en base a los resultados obtenidos, se podrán ajustar los tipos y cantidades de variables en la medida de lo necesario para producir y comparar resultados alternos.

Este módulo estará construido utilizando las librerías del motor de Weka (Waikato Environment for Knowledge Analysis - Entorno para Análisis del Conocimiento de la Universidad de Waikato) [11], el cual es un conocido software para aprendizaje automático y minería de datos escrito en Java. WEKA es un software libre distribuido bajo licencia GNU GPL y dado que Einstein se está desarrollando totalmente sobre el lenguaje Java, se logra una integración natural y transparente en el producto final, pudiendo ejecutarse en cualquier plataforma que tenga el JRE de Java (Java Runtime Environment) versión 1.5 o superior.

El segundo componente de este módulo es el Sistema Experto, el cual es el encargado de realizar la evaluación final de los grupos significativos revelados por el componente de Minería de Datos.

Será construido utilizando Drools, un sistema de administración de reglas de negocio (BRMS) con un motor de reglas basado en una adaptación orientada a objetos del algoritmo Rete[12]. 
Drools permite expresar de una forma más natural las reglas de diagnóstico, interactuando con los objetos propios del sistema. Provee separación de lógica (reglas) y datos (hechos). También aporta soporte para la programación declarativa, y es lo suficientemente flexible para expresar la semántica del problema con un lenguaje específico de dominio (DSL). Drools utiliza el lenguaje de reglas de drools (DRL) para especificar las condiciones, acciones y funciones de las mismas, las cuales se pueden expresar con distintos lenguajes, como Java y MVEL. Luego las reglas serán guardadas en archivos de texto con la extensión drl.

De esta manera las reglas de diagnóstico, que normalmente son muy cambiantes, podrán ser ajustadas de una manera externa al sistema sin necesidad de rediseñar, modificar y redistribuir el sistema[13].

\section{Módulo de Reportes}

En el se desplegarán los resultados generados a partir de la aplicación de las reglas. Se visualizarán las recomendaciones para un grupo de individuos, para un individuo en particular y en forma optativa las reglas aplicadas.

Se visualizarán los parámetros del agrupamiento generado por el algoritmo de Minería de Datos, como características socioeconómicas de cada grupo en relación con los resultados de la evaluación.

Individualmente, se podrán ver las respuestas generadas y su comparación con las respuestas correctas de un estudiante en particular, junto con sus datos socioeconómicos.

En virtud de la portabilidad de los reportes generados, el módulo permitirá utilizar diversos formatos estándar.

\section{Resultados preliminares}

Para el análisis preliminar de las respuestas de los alumnos se empleó el algoritmo de conglomerados en dos fases (segmentación bietápica o two step).

Este procedimiento es factible en colecciones de datos que requieren un tratamiento de variables categóricas y continuas a la vez. Al suponer que las variables son independientes, es posible aplicar una distribución normal multinomial conjunta en las variables continuas y categóricas.

Mediante la comparación de los valores de un criterio de selección del modelo para diferentes soluciones de conglomeración, el procedimiento determina automáticamente el número óptimo de conglomerados y permite alta escalabilidad cuando se trata con un alto volumen de datos, mediante la construcción de un árbol 
de características de conglomerados. Adicionalmente presenta la ventaja de ser bastante robusto frente al incumplimiento de alguna de las hipótesis.

La muestra analizada está conformada por 32 alumnos de primer año de una universidad privada. Se relevaron dos tipos de variables: el primero relacionado con aspectos socioeconómicos (edad, sexo, número de horas semanales de trabajo, quién costea los estudios, tipo de título secundario obtenido, etc.) con el objetivo de analizar aquellos aspectos que podrían incidir en el rendimiento académico de los alumnos.

El segundo grupo de variables está constituido por las respuestas de los alumnos a 10 ejercicios sobre matrices, determinantes y sistemas de ecuaciones lineales de ecuaciones propuestos a modo de evaluación. Con el fin de identificar las áreas en que se presentan las mayores dificultades en relación a estos contenidos, los ejercicios fueron agrupados en tres categorías, según el tipo de actividad que demanda su resolución: Algebraicos (sólo requieren la aplicación de un procedimiento de carácter algorítmico), Conceptuales (Requieren el conocimiento y dominio de los conceptos involucrados e implican un mayor nivel de abstracción) y de Modelización (suponen el reconocimiento de los contenidos como herramienta adecuada para resolver una situación o problema concretos y el planteo en un marco algebraico del problema a resolver).

Si bien la hipótesis de normalidad no se verifica para las variables "Edad" y "Número de horas de trabajo semanales", como se mencionó anteriormente, el algoritmo resulta bastante robusto frente a violaciones de las distribuciones [15]

Como puede observarse en la Fig. 3, la calidad de los conglomerados resulta buena. El algoritmo clasificó los datos en dos conglomerados como se muestra en la Fig.3 y Fig. 4

Resumen de modelo

\begin{tabular}{|l|l|}
\hline Algoritmo & Bietápico \\
\hline Entradas & 8 \\
\hline Conglomerados & 2 \\
\hline
\end{tabular}

Calidad de conglomerado

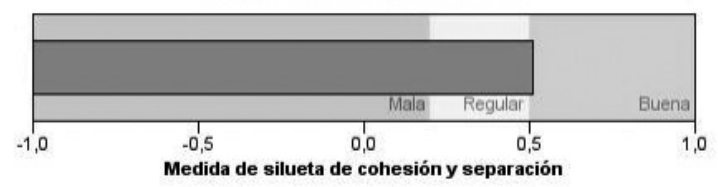

Fig. 3: Resumen del modelo 
Tamaños de conglomerados

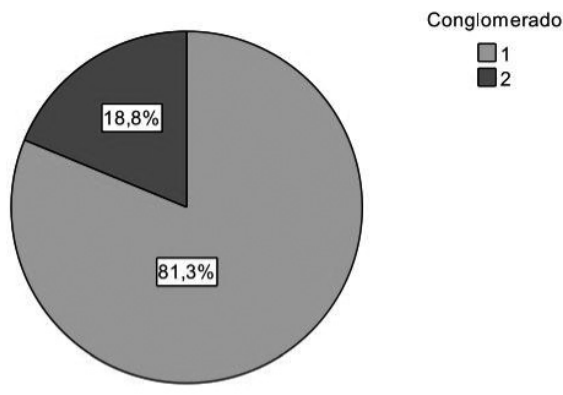

\begin{tabular}{|l|l|}
\hline $\begin{array}{l}\text { Tamaño de } \\
\text { conglomerado más } \\
\text { pequeño }\end{array}$ & $6(18,8 \%)$ \\
\hline $\begin{array}{l}\text { Tamaño de } \\
\text { conglomerado más } \\
\text { grande }\end{array}$ & $26(81,2 \%)$ \\
\hline $\begin{array}{l}\text { Cociente de tamaños: } \\
\text { Conglomerado más } \\
\text { grande a conglomerado } \\
\text { más pequeño }\end{array}$ & 4,33 \\
\hline
\end{tabular}

Fig. 4: Tamaño de los conglomerados

\section{A. Caraterísticas de los conglomerados}

La Tabla III muestra las características más relevantes de cada conglomerado cuando se tienen en cuenta las variables socioeconómicas del primer grupo y sólo la variable "Nota" correspondiente al resultado final de la evaluación.

TABLA III: Características de los Conglomerados

\begin{tabular}{|l|l|l|}
\hline VARIABLE & CONGLOMERADO 1 & CONGLOMERADO 2 \\
\hline Edad promedio & 19.73 años & 24 años \\
\hline Horas de trabajo semanal & 1.92 & 32.33 \\
\hline Costeo de estudios & Padres $(92.3 \%)$ & Propio $(83.3 \%)$ \\
\hline Turno más frecuente & Mañana $(88.5 \%)$ & Noche $(100 \%)$ \\
\hline Título más frecuente & Bachiller $(42.3 \%)$ & Técnico $(66.7 \%)$ \\
\hline Nota promedio & $3.31($ Variable relativa $50.4 \%)$ & 3 (Variable relativa $55.8 \%)$ \\
\hline Sexo & Masculino $(73.1 \%)$ & $100 \%($ Masculino) \\
\hline Proviene de otra universidad & No $(88.5 \%)$ & No $(66.7 \%)$ \\
\hline
\end{tabular}


En el "Conglomerado 1" se agrupan los casos de alumnos con menor cantidad de horas de trabajo semanales, siendo la media de 1.92 horas y con un promedio de edad de 19,73 años. El promedio de edades de los casos agrupados en este conglomerado es significativamente menor que la edad promedio de los alumnos agrupados en el Conglomerado 2.

De estos alumnos, un $88.5 \%$ cursa en el turno mañana y en el $92.3 \%$ de los casos los estudios son costeados por sus padres. El $42.3 \%$ poseen título de "bachiller".

La nota promedio de la evaluación es de 3.31 puntos con una variabilidad relativa del $50.4 \%$

En el "Conglomerado 2" la variable de mayor relevancia es el "turno en el que cursan"; el 100\% de estos alumnos cursan en el turno noche.

E1 $83.3 \%$ de estos alumnos costea sus propios estudios y trabajan, en promedio 32.33 horas semanales.

El promedio de edades de estos alumnos es de 24 años y el $66.7 \%$ posee título secundario de técnico.

En este conglomerado el promedio de notas en la evaluación implementada es de 3 puntos con una variabilidad relativa del $55.8 \%$

El "conglomerado 1" presenta una nota promedio levemente superior al promedio de notas del "Conglomerado 2" y con una menor variabilidad relativa.

En ambos conglomerados se observa un rendimiento bajo en relación a la puntuación obtenida en la evaluación, con una alta variabilidad.

Si bien la muestra es muy pequeña, en una primera aproximación, podría inferirse que los casos agrupados en el "Conglomerado 1" manifiestan el comportamiento típico en relación a las variables estudiadas ya que poseen una mayor similitud con el total de la muestra, mientras que los casos agrupados en el "Conglomerado 2" manifiestan mayor diferencia en relación al conjunto global de datos.

Si se analizan los datos discriminados según la categorización "ejercicios algebraicos, conceptuales y de modelización" se observa que si bien no se presentan diferencias significativas en ninguno de los dos conglomerados en comparación con la muestra global, en el "Conglomerado 1" se agrupan los alumnos con un desempeño levemente superior en las áreas Conceptual y de Modelización.

En el Conglomerado 2 se ubican aquellos que tuvieron mejor desempeño en el área Algebraica con una puntuación promedio de 2 sobre un total de 4 puntos y una variabilidad muy similar a la del Conglomerado 1 y a la muestra global, como puede observarse en la Tabla IV. 
Tabla IV: Resumen por Categoría

\begin{tabular}{|l|l|l|}
\hline VARIABLE & - & CONGLOMERADO 2 \\
\hline Ejercicios algebraicos & $\begin{array}{l}\text { Regular: } 46.2 \% \\
\text { Malo: } 36.61 \% \\
\text { Buenos: } 19.23 \%\end{array}$ & $\begin{array}{l}\text { Regular: } 33.3 \% \\
\text { Malo: 33.3\% } \\
\text { Buenos: } 33.33 \%\end{array}$ \\
\hline Ejercicios conceptuales & $\begin{array}{l}\text { Regular: } 42.3 \% \\
\text { Malo: } 42.3 \%\end{array}$ & $\begin{array}{l}\text { Malo: } 88.3 \% \\
\text { Regular: } 11.7 \% \\
\text { Buenos: } 0 \%\end{array}$ \\
\hline Ejercicios de modelización & $\begin{array}{l}\text { Malo: } 92.3 \% \\
\text { Regular: } 7.3 \%\end{array}$ & Malo $100 \%$ \\
& Buenos: $0 \%$ & \\
\hline
\end{tabular}

\section{Conclusión}

Se ha finalizado el desarrollo de los tres componentes del Módulo Origen de Datos, se ha implementado una primera evaluación consistente en una serie de problemas referidos a temas de Álgebra Lineal y se efectuó la captura de datos mediante Limesurvey, obteniendo un total de 32 encuestados.

El proyecto se encuentra en estos momentos en el desarrollo del componente de Minería de Datos y del Módulo Inteligente los cuales permiten, no sólo detectar patrones y construir los perfiles de alumnos con características similares, necesarios para elaborar las reglas del Sistema Experto, sino que brinda la posibilidad de poner a prueba el Módulo Origen de Datos y realizar los ajustes y correcciones necesarios en los componentes de dicho módulo. También permiten conocer si el tipo de respuestas incorporadas en el módulo Evaluación de Resultados es la más adecuada para los objetivos del proyecto o si resulta necesario incorporar otras alternativas. Asimismo se espera que brinde información acerca de la pertinencia de los ejercicios planteados en esta primera evaluación en función de los objetivos del proyecto.

A partir de la recopilación y análisis de datos se espera poder realizar una primera aproximación que permita tipificar los errores en las producciones de los alumnos lo cual serviría en primer lugar para desarrollar las futuras evaluaciones $\mathrm{y}$, en segundo lugar, permitiría ir perfeccionando progresivamente el sistema en función de los resultados que se vayan obteniendo.

Con el escaso volumen de datos obtenidos hasta el momento no es posible establecer ninguna tendencia. Sin embargo, la diferencia visualizada en el desempeño académico respecto de los ejercicios de modelización refleja cierta 
dificultad en la asimilación de los conceptos de modelizado. También se observa un bajo rendimiento generalizado.

Queda pendiente el desarrollo de evaluaciones dinámicas que, a partir de la modificación de distintos parámetros, permitan la creación de diversos conjuntos de problemas y ejercicios para un mismo contenido.

\section{Referencias}

[1] Camilloni A. et al, ¿Qué significa evaluar? En La evaluación de los aprendizajes en el debate didáctico contemporáneo, pp. 35-66. Paidós (1998)

[2] Vílchez Quesada, E.: Sistemas Expertos para la enseñanza y el aprendizaje de la Matemática en la Educación Superior. CIEMAC (2007)

[3] Theory of didactical situations in Mathematics.G. Brousseau.pp 30-31

[4] Sun Trademark and Logo Usage Requirements, http://www.sun.com/policies/ trademarks

[5] Erich Gamma, et al. "Design Patterns: Elements of Reusable Object-Oriented Software". pp 315. Addison-Wesley. (1995)

[6] Lime Survey, http://www.limesurvey.org/

[7] Senso, J.A., El concepto de metadato. Algo más que descripción de recursos electrónicos, Ci. Inf., 32, pp. 95-106 (2003)

[8] YAML: a human friendly data serialization standard for all programming languages, http://www.yaml.org/

[9] UCLA Academic Technology Service, http://www.ats.ucla.edu/stat/spss

[10] Johnson, R., Wichern, D., Applied Multivariate Statistical Analysis,pp. 690691, Prentice Hall (2002)

[11] WEKA,http://www.cs.waikato.ac.nz/ml/weka/Forgy C.

[12] Drools,http://www.jboss.org/drools/documentation.html

[13] Forgy C., Rete: A Fast Algorithm for the Many Pattern/Many Object Pattern

[14] Match Problem, Artificial Intelligence, 19 (1982)

[15] IBM Corporation, IBM SPSS Statistics Base 20. Pag. 171 (2011) 
